\title{
ARTÍCULOS LIBRES
}

\section{EL "RING" DEL BANCO MUNDIAL: ESTRATEGIAS DISCURSIVAS EN EL ENFRENTAMIENTO DE LA ECONOMÍA}

Y LA SOCIOLOGÍA PARA LA MEDICIÓN DE LA DESIGUALDAD Y MOVILIDAD SOCIAL'

\section{THE "RING" OF THE WORLD BANK: DISCURSIVE STRAIEGIES IN THE CONFRONTAIION OF THE ECONOMY AND SOCIOLOGY FOR THE MEASUREMENT OF INEQUALITY AND SOCIAL MOBILITY}

VICTORIA MATOZO 2

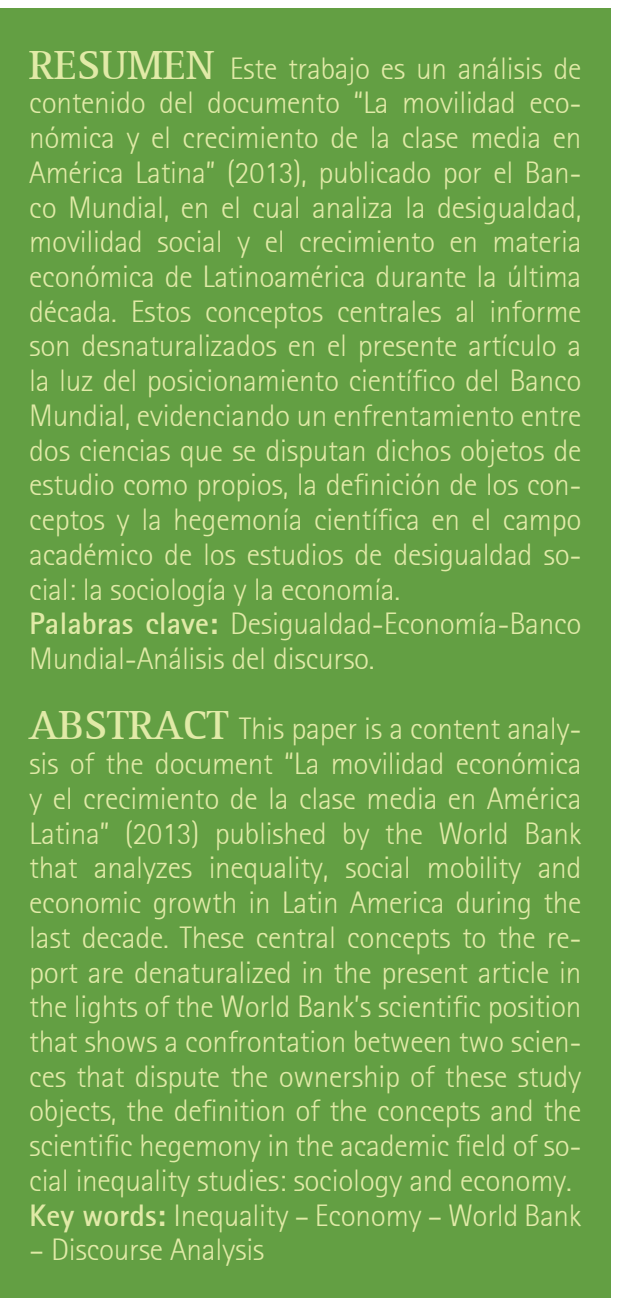

1 Trabajo recibido el 25 de octubre de 2017 y aprobado el 15 de agosto de 2018.

${ }^{2}$ Pertenencia Institucional: Instituto de Investigaciones Gino Germani. Becaria doctoral Conicet. Instituto de lnvestigaciones Gino Germani, Facultad de Ciencias Sociales, UBA-Conicet.

\section{INTRODUCCIÓN}

Este trabajo pretende realizar un análisis y una reflexión sobre la medición de desigualdades dentro del marco de los estudios de movilidad social realizados por organismos internacionales, aquellos mismos que generan recomendaciones y financian politicas sociales para América Latina a partir de las evaluaciones que realizan sobre nuestras sociedades. Más especificamente, aqui se estudia el documento "La movilidad económica y el crecimiento de la clase media en América Latina" publicado en 2013 por el Banco Mundial, una obra que se presenta como el examen de las tendencias y los cambios en la movilidad social de la población y el crecimiento económico de la región en la última década.

El Banco Mundial se propone como un organismo especializado de las Naciones Unidas (ONU) que evalúa, financia y asiste técnicamente a paises en desarrollo en búsqueda de acabar con la pobreza extrema y fomentar la prosperidad compartida. El informe a analizar surge en un momento histórico en el cual diferentes organismos internacionales, como el Fondo Monetario Internacional, la Organización Mundial del Comercio, la Organización para la Cooperación y el Desarrollo Económico o el Banco Mundial, realizan distintos estudios en Latinoamérica sobre desigualdad, pobreza y temas afines con el objetivo de recetar políticas públicas o vías de acción para mejorar la situación en la región; dado que dichas instituciones son quienes financian estos cambios y colaboran económicamente con el establecimiento de políticas de crecimiento o realizando préstamos para que los paises puedan superar los problemas de sus balanzas de pagos. Este financiamiento, sin embargo, es realizado una vez que el organismo internacional en cuestión analiza la situación del Estado a financiar en relación con el acatamiento de las recomendaciones generadas o las políticas públicas propuestas. Nuestro interés se centra en desgranar este documento no en cuanto a evaluación, sino evidenciar qué categorias se utilizan para la medición de desigualdades y movilidad social, y a qué paradigma teórico responden, y estudiar el posicionamiento político que el Banco Mundial tiene sobre estos temas. 
De Prácticas y discursos

Universidad Nacional del Nordeste

Centro de Estudios Sociales

Año 7, Número 10, 2018, Octubre

ISSN 2250-6942

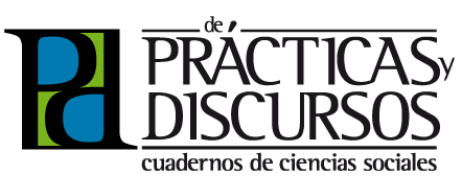

Nuestra clave de lectura estará marcada por la hipótesis de que los informes de organismos internacionales en materia de desigualdades y estructura social utilizan sistemas de indicadores sociales, conceptos y formas de medición propias de las Ciencias Económicas, desconociendo los aportes que la sociología ha realizado a este campo de estudio. Si bien la discusión entre economía y sociología no es nueva, nos interesa indagar cómo la misma se desarrolla dentro del discurso del Banco Mundial. Es menester aclarar que la discusión a analizar será el enfrentamiento entre dos ciencias sin indagar los matices que podemos encontrar al interior de ambas, ya que por ejemplo no será lo mismo el concepto de desigualdad para economistas marxistas que para economistas neoliberales, nos interesa la justificación disciplinar. Y dentro de la misma, exponer la posición del Banco Mundial, y no desarrollar en profundidad la posición de la sociología o la economía como ciencia en el debate suscitado, ya que nuestro objetivo es abordar el "enfrentamiento" de ambas disciplinas a partir de la perspectiva que esta institución toma en su informe.

En este aspecto es necesario contextualizar la aparición de esta publicación, ubicarla históricamente y rastrear el rol del Banco Mundial, junto a otros organismos internacionales que generan recomendaciones a los Estados, en la economía y la política de América Latina. Luego de la década de 1980, llamada la "década pérdida" por la Comisión Económica para América Latina y el Caribe (Cepal, 1996) a causa de que la economía no crecía, numerosos conflictos en materia económica sacudian a Latinoamérica. En el caso de la Argentina, el estallido de la hiperinflación de 1989 condujo al llamado de elecciones anticipadas. Por esos años, el economista John Williamson presenta el Consenso de Washington en 1989 (cfr. Williamson, 2003), un conjunto de diez medidas para paises en desarrollo azotados por la crisis económica del momento, las cuales formaban parte de la agenda de discusión de organismos económicos internacionales como el Fondo Monetario Internacional (FMI) y el Banco Mundial (BM). Frente al fracaso de la intervención estatal en la economía evidenciado en Argentina por la hiperinflación, estas recomendaciones neoliberales, que proponían al sector privado como motor de la economía, la liberación del mercado y la apertura como estrategia de inserción en la economía mundial, fueron adoptadas en varios paises de Latinoamérica. En Argentina, las medidas fueron adoptadas durante la década de 1990 como salida a la hiperinflación, avalando un diag- 
De Prácticas y discursos

Universidad Nacional del Nordeste

Centro de Estudios Sociales

Año 7, Número 10, 2018, Octubre

ISSN 2250-6942 el "RING" Del banco MUNDIAL: ESTRATEGIAS DISCURSIVAS en el enFrentaMIENTO DE LA ECONOMÍA Y La SOCIOLOGİA PARA LA MEDICIŌN DE LA DESIGUALDAD Y MOVILIDAD SOCIAL

THE "RING" OF THE WORLD BANK: DISCURSIVE STRATEGIES IN THE CONFRONTAIION OF THE ECONOMY AND SOCIOLGGY FOR THE MEASUREMENT OF INEQUALIITY AND SOCIAL MOBILITY

nóstico económico que caracterizaba al intervencionismo estatal de épocas anteriores como ineficientes.

Estas políticas adoptadas durante la década de 1990 tuvieron efectos sociales de aumento de pobreza y desigualdad social, entre otros, que comenzaron a poner en duda la eficiencia de las recomendaciones, desde la economía como ciencia y desde la política como práctica. Muchas críticas surgieron desde la economía durante ese período y en los últimos años, críticas que por ejemplo plantean que los países centrales productores de las "recetas neoliberales" no permiten a los países en desarrollo aplicar las políticas que ellos mismos adoptaron para poder llegar a ser potencias mundiales (políticas proteccionistas e intervencionistas incluso), "quitando la escalera" por la cual ellos alcanzaron el desarrollo económico (cfr. Chang, 2004). Asimismo, algunos premios nobel han criticado duramente la actuación del FMI y el BM con estas recomendaciones. Es el caso de Joseph Stiglitz, Premio Nobel de Economía (2001) y ex vicepresidente y economista en jefe del Banco Mundial (1997-2000), quien en numerosas ocasiones denunció el interés de organismos como el FMI en fortalecer a Estados Unidos en detrimento de los paises tercermundistas y cómo la globalización arruinó a varios paises emergentes, con algunas excepciones como, por ejemplo, China, que se salvó gracias a no seguir las recomendaciones de estos organismos (Cfr. Stiglitz, 2012). Con relación a nuestro país, varias veces Stiglitz reivindicó el rumbo de la economía argentina bajo el kirchnerismo y su negación a seguir las recomendaciones del FMI.

Desde la práctica política, el fortalecimiento del Mercosur, los llamados "nuevos populismos en América Latina" del siglo XXI (Lula Da Silva y Dilma Roussef en el Brasil, Evo Morales en Bolivia, Hugo Chávez en Venezuela, Néstor y Cristina Kirchner en la Argentina, Rafael Correa en el Ecuador, etc.) partidarios de políticas económicas proteccionistas y el movimiento "No al Alca" (movimiento en contra del Área de Libre Comercio de las Américas) de 2005, entre otras situaciones políticas relevantes en la región, han puesto de manifiesto el rechazo a modelos y recomendaciones provenientes de las grandes potencias yos organismos internacionales con sede en ellas.

Frente a este panorama crítico, los organismos internacionales han tenido que reposicionarse para que sus recomendaciones sigan teniendo valor en la región. Por estas razones es que consideremos que los discursos del FMI y el BM han tenido un estilo 
De Prácticas y discursos

Universidad Nacional del Nordeste

Centro de Estudios Sociales

Año 7, Número 10, 2018, Octubre

ISSN 2250-6942

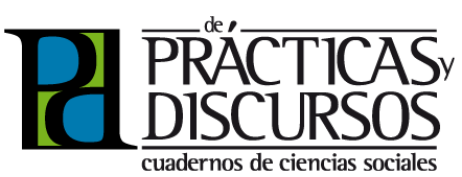

más abierto y conciliador en los últimos años en cuanto a las recomendaciones planteadas, y han reconocido otras perspectivas teóricas para analizar desigualdades, a fin conciliar intereses con los Estados latinoamericanos y poder trabajar en conjunto.

Dentro de este contexto es que se publica el documento que analizaremos en este trabajo, en el que nos centraremos no en reponer esta perspectiva histórica, sino en desentrañar la perspectiva teórica que este informe posee, a partir de su conceptualización de los paradigmas enfrentados en la medición de desigualdades sociales y de la definición, elección y justificación de perspectivas teóricas, categorias y unidades de análisis del documento, abordando las elecciones que el Banco Mundial realizó al definir conceptos tan amplios como el de movilidad social, llevando a cabo un análisis del discurso de dicho documento. A grandes rasgos, los conceptos que el informe maneja se nos presentan como problemáticos ya que son "significantes flotantes" (Laclau, 1995; Zizek, 1992; Lacan, 1988) cuyo significado está sobredeterminado (es polisémico, posee significados múltiples) y cuya identidad depende de la articulación con otros significantes, otros términos y conceptos que le servirán de marco para definirse a sí mismo. Ese marco dentro del cual se definen los conceptos utilizados en el informe será nuestro objeto de estudio. Dada la longitud del documento y la gran variedad de temas que analiza, examinaremos el primer apartado del documento denominado Panorámica General, que expone las bases, concepciones y categorizaciones de movilidad social y clase media, donde se plasman los objetivos y las perspectivas analíticas que entran en tensión y que elige el informe, y se definen y justifican algunos conceptos utilizados en el análisis, y las formas de medir y definir dichas categorias.

\section{PRODUCTORES DE SENTIDO: GÉNERO Y DISCURSO}

En primer lugar, es menester aclarar que estamos analizando un producto, un discurso desde su materialidad y desde su producción. Dentro de los análisis del discurso existe una primera gran clasificación que organiza metodológicamente la tarea, entre análisis en producción y análisis en reconocimiento (los estudios de recepción). En este trabajo realizaremos un análisis en producción que, sin embargo, no estará ajeno a la recepción del discurso ya que el sistema productivo del sentido se compone de 
De Prácticas y discursos

Universidad Nacional del Nordeste

Centro de Estudios Sociales

Año 7, Número 10, 2018, Octubre

ISSN 2250-6942 el "RING" Del banco MUNDIAL: ESTRATEGIAS DISCURSIVAS en el enFrentaMIENTO DE LA ECONOMÍA Y LA SOCIOLOGía PARA LA MEDICIŌN DE LA DESIGUALDAD Y MOVILIDAD SOCIAL

THE "RING" OF THE WORLD BANK: DISCURSIVE STRATEGIES IN THE CONFRONTAIION OF THE ECONOMY AND SOCIOLGGY FOR THE MEASUREMENT OF INEQUALIITY AND SOCIAL MOBILITY

la articulación entre ambos momentos (Verón, 1988).El análisis "en producción" que proponemos sigue el razonamiento de Eliseo Verón, que afirma que:

El análisis del discurso consiste en la referencia, en la superficie discursiva, de las huellas que conducen a las condiciones de producción de los discursos. Estas huellas son el soporte de operaciones que se deben reconstruir, operaciones que toman la forma de reglas de generación de estos discursos. (Verón, 1988:201)

Estas "huellas" serán las palabras, los términos y conceptos centrales a definir, los significantes flotantes de los que hablábamos, las marcas del enunciador y otros procedimientos retóricos que generan efectos de sentido y dejan rastros del proceso productivo y las operaciones realizadas para crear el discurso-producto.

En segundo lugar, analizaremos el enunciado, como unidad mínima del discurso, siguiendo la postura de Michael Bajtín en tres momentos: el contenido temático, el estilo y la estructuración del mismo. La unión indivisible de estos tres momentos es lo que Bajtín denomina géneros discursivos, que son "tipos relativamente estables de enunciados" (Bajtín, 1979:248). Dentro de la heterogeneidad de los géneros podemos afirmar que el corpus que analizamos pertenece a un género secundario, producido en situaciones complejas de comunicación, organizadas y desarrolladas, que se presenta de forma escrita: el informe de investigación.

El primer momento planteado por Bajtín es el del contenido, el recorte temático específico del informe de investigación. El contenido del discurso nos conduce a definir cuál es la lógica isotópica del discurso. Este informe se inserta en el contexto de los estudios sobre estructura social. Internamente el discurso versa sobre este campo que, a su vez, está entrecruzado por los campos de estudio de la economía y de la sociología que se ocupan de la misma unidad de discurso pero de formas muy diferentes. Esa diferencia es también parte de un campo semántico, en el cual se entrecruzan, discuten y se enfrentan ambas disciplinas. La repetición que realiza el informe del Banco Mundial al comienzo da muestra de lo que estamos diciendo: en las primeras páginas se explicita el objeto del informe, la clase media y la movilidad social en Latinoamérica, y se exponen los dos campos que definen estos conceptos, la sociología y la economía, realizando 
una caracterización de las categorías utilizadas y la diferencia de ambas ciencias en torno a dicha cuestión, con el fin de que el lector/destinatario pueda discernir la temática de la cual ese habla. Esta lógica isotópica le da coherencia interna al discurso y es una clave de lectura para el destinatario, es una propiedad semántica del texto.

El informe entonces tiene la lógica isotópica del encuentro de estas tres categorías semánticas, estos tres campos (remarcado en azul), mientras que los capítulos siguientes del informe se centrarán en los Estudios sobre la estructura social realizados por el campo de la economía (remarcado en rojo):

Campos semánticos del Informe "La movilidad económica y el crecimiento de la clase media en América Latina" (Banco Mundial, 2013)

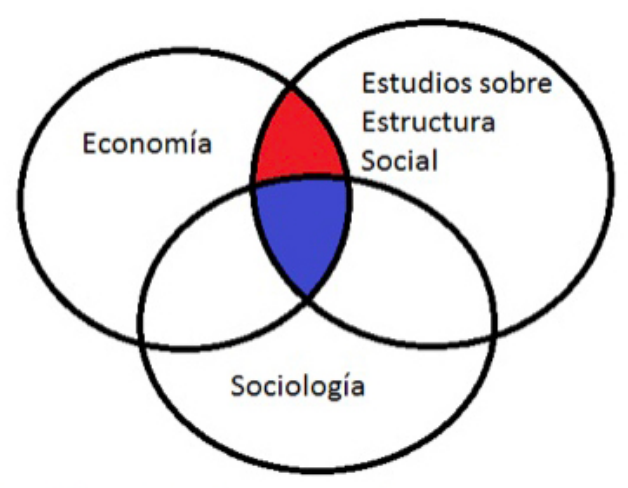

Fuente: Cuadro de creación propia.

Siguiendo la conceptualización de Pierre Bourdieu (Contursi y Ferro, 2000:8), podemos decir que los tres campos nombrados son parte del campo académico que los engloba y dentro del cual se dan luchas de poder entre el campo de la sociología y el de la economía. Esta lucha se realiza de forma sutil exponiendo el hábitus de los agentes que en ese campo luchan entablando una conversación en la que el enunciador intentará convencer a un lector que reconoce como par, miembro de una comunidad de académicos o intelectuales. Esta forma de la lucha expone el segundo momento del enunciado, que se refiere al estilo. Los recursos léxicos, gramaticales y fraseológicos desplegados en el texto se acercan al lenguaje académico propio del género informe, al igual que la relación social de carácter formal entre enunciador y enunciatario dentro del texto, como evidenciamos en una amplia referencia al estado de la cuestión realizando comparaciones de perspectivas 
De Prácticas y discursos

Universidad Nacional del Nordeste

Centro de Estudios Sociales

Año 7, Número 10, 2018, Octubre

ISSN 2250-6942 el "RING" Del banco MUNDIAL: ESTRATEGIAS DISCURSIVAS en el enFrentaMIENTO DE LA ECONOMÍA Y La SOCIOLOGİA PARA LA MEDICIŌN DE LA DESIGUALDAD Y MOVILIDAD SOCIAL

THE "RING" OF THE WORLD BANK: DISCURSIVE STRATEGIES IN THE CONFRONTAIION OF THE ECONOMY AND SOCIOLGGY FOR THE MEASUREMENT OF INEQUALIITY AND SOCIAL MOBILITY

teóricas (entre economía y sociología), la adopción de un modo de análisis (elección de medida, variables, definiciones y perspectiva teórica), la referencia a un marco histórico, las referencias a autores centrales en los estudios de movilidad social (en el apartado de Conceptos y Medidas se hace referencia a Max Weber, Carl Marx, Erikson y Goldthorpe, etc.) y otras marcas textuales que definen el estilo académico del texto.

Finalmente analizaremos el tercer momento del enunciado, que se refiere a la estructuración del discurso. El género informe de investigación aqui analizado organiza sus enunciados con una secuencia lógica de presentación de un problema cognitivo, resolución analítica y evaluación del mismo. Pero antes de avanzar en esta secuencia de tres momentos y habiendo definido el género del discurso que estamos analizando, creemos pertinente definir el tipo de discurso.

\section{TODO INFORME ES POLÍTICO}

Ya establecimos el género informe para el discurso analizado, pero dentro de la tipología de discursos podemos decir que el informe analizado pertenece al discurso político en el sentido de que es producido por una institución política (Verón, 1987), en primer lugar. Y, en segundo lugar, el informe analizado tiene una dimensión política desde su función, que se sigue de la función misma del Banco Mundial en términos de sus recomendaciones e intervenciones en paises latinoamericanos. Siendo un organismo de financiamiento, los Estados deben amoldarse a dichas recomendaciones para que diversos proyectos sean financiados por la institución internacional. Esta cualidad le da al informe un carácter político, ya que su lectura no será meramente informativa por parte de los gobiernos latinoamericanos.

En el plano del enunciado podemos identificar, siguiendo a Verón (1987), un primer nivel, el de los destinatarios que analizaremos más adelante, y un segundo nivel, de las entidades y los componentes del discurso político: descriptivo, didáctico, prescriptivo y programático. Este segundo nivel es el que analizaremos conjuntamente con la estructuración del género informe de investigación.

Las entidades presentes en este texto son principalmente la clase media y la pobreza, entidades que en otros discursos podian aparecer como "formas nominales" (Verón, 1987:6) que poseen un poder explicativo y son operadores de interpretación, pero en 
De Prácticas y discursos

Universidad Nacional del Nordeste

Centro de Estudios Sociales

Año 7, Número 10, 2018, Octubre

ISSN 2250-6942

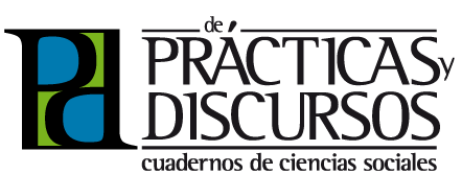

este informe la clase media y la pobreza son categorías problematizadas, definidas desde diferentes perspectivas y apropiadas bajo el discurso economista luego de una reposición del estado de la cuestión y una posición teórica y técnica que justifica su utilización para la medición que el informe realiza. En este caso, entonces, estas categorias son entidades enumerables más amplias que los colectivos de identificación, "que designan colectivos que no funcionan como operadores de identificación de los actores en presencia" (Verón, 1987:5), están en disputa y funcionan de forma diferente en otros tipos de discurso.

Otras entidades presentes en el texto son los colectivos "economistas" y "sociólogos", que conforman las dos perspectivas epistemológicas enfrentadas en el texto. Estas son también entidades enumerables que no se identifican con ningún actor presente en el discurso, no crean un "nosotros" inclusivo ni tampoco un "ellos" adversario, sino que son identidades asociadas al paradestinatario (que será analizado más adelante) a quien el enunciador intentará persuadir. Siendo que nuestra clave de lectura es indagar la posición epistemológica del Banco Mundial en cuanto a la investigación en desigualdades y estructura social, estas entidades son centrales en nuestro trabajo de rastrear la posición del enunciador. Al respecto, el texto afirma: "A pesar de que en los siguientes capítulos aparecen a menudo los enfoques sociológicos, el informe analiza la movilidad y la dinámica de clase fundamentalmente desde una perspectiva económica" (Ferreira y otros, 2013:28).

Si bien se plantea una perspectiva económica en el informe, en ningún momento el enunciador se identifica con el colectivo "economistas", no toma posiciones a favor o en contra de ninguno de los colectivos en disputa y reconoce "enfoques sociológicos" en su explicación. El distanciamiento con la entidad podría estar relacionado con el objetivo del Banco Mundial y otros organismos internacionales de alejarse de una perspectiva económica de corte neoliberal que, como explicamos en la introducción, fue un paradigma característico de los 90 en estos organismos y es duramente criticado hoy, incluso por economistas que pertenecieron a estas instituciones.

Para avanzar sobre el análisis de los componentes del discurso político, retomaremos el tercer momento de los géneros propuesto por Bajtín, la estructuración del género que en este caso es la secuencia lógica del género informe de investigación: pre- 
De Prácticas y discursos

Universidad Nacional del Nordeste

Centro de Estudios Sociales

Año 7, Número 10, 2018, Octubre

ISSN 2250-6942 el "RING" Del banco MUNDIAL: ESTRATEGIAS DISCURSIVAS en el enFrentaMIENTO DE LA ECONOMÍA Y LA SOCIOLOGÍA PARA LA MEDICIÓN DE LA DESIGUALDAD Y MOVILIDAD SOCIAL

THE "RING" OF THE WORLD BANK: DISCURSIVE STRATEGIES IN THE CONFRONTAIION OF THE ECONOMY AND SOCIOLGGY FOR THE MEASUREMENT OF INEQUALIITY AND SOCIAL MOBILITY

sentación de un problema cognitivo, resolución analítica y evaluación del mismo.

El primer momento de esta secuencia, la presentación del problema, se expone en las bases textuales del informe descritas por Egon Werlich (Contursi y Ferro, 2000, unidades estructurales que funcionan como inicio del texto. En este caso, el informe comienza afirmando:

En este informe se abordan los principales conceptos relativos a la movilidad social y se documentan las principales tendencias y hechos estilizados en América Latina y el Caribe en los últimos decenios, realizando comparaciones tanto entre generaciones como a lo largo del ciclo vital. (Ferreira y otros, 2013:19)

Aquí el texto expone su base temática, los conceptos relacionados a movilidad social y la secuencia que comenzará con las principales tendencias y hechos en Latinoamérica. Este primer momento de presentación de un problema cognitivo se hace presente en palabras y frases como "en este informe se abordan", "se plantea", "se presenta", y otros términos similares que además ofrecen al lector una pauta de lectura: el discurso es un panorama, una descripción, presentación, que se presenta como objetiva y académica (escrita por académicos) con frases como "este estudio investiga", "este estudio documenta", "la evidencia presentada en el informe", etc. En las citas anteriores y durante toda la presentación del problema cognitivo abunda el componente descriptivo:

Tras décadas de estancamiento, la población de clase media en América Latina y el Caribe ha aumentado en un 50\% -de 103 millones de personas en 2003 a 152 millones (o un 30\% de la población del continente) en 2009. (Ferreira y otros, 2013:19)

En este apartado, por ejemplo, se describe una situación en pasado ("ha aumentado" pretérito perfecto compuesto) siguiendo la lógica de un mundo narrado (cfr. Weinreich, 1975) que señalaremos más adelante. En la presentación también se expone la discusión que le da nombre a este trabajo, el enfrentamiento epistemológico entre economía y sociología en el campo de los estudios sobre estratificación social:

Definir la clase media no es un asunto trivial, y las alternativas dependen de la perspectiva del investigador. Por ejemplo, los sociólogos y los politólogos suelen definir la clase media en términos del nivel educativo (por ejemplo, un nivel de estudios su- 
De Prácticas y discursos

Universidad Nacional del Nordeste

Centro de Estudios Sociales

Año 7, Número 10, 2018, Octubre

ISSN 2250-6942

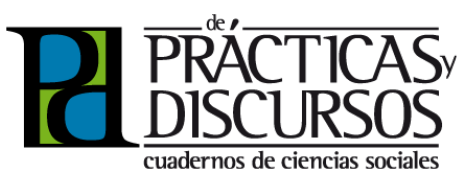

perior a la secundaria), del empleo (normalmente no manual) o de la propiedad de activos (que a su vez incluye la propiedad de bienes básicos duraderos o de una vivienda). Los economistas, en cambio, tienden a centrarse en el nivel de ingresos. Este estudio adopta una perspectiva económica, pero para llegar a una definición más robusta -menos arbitraria-, ancla la definición basada en los ingresos en el concepto fundamental de seguridad económica (entendida como una baja probabilidad de volver a caer en la pobreza). (Ferreira y otros, 2013:19)

En primer lugar, este párrafo se refiere a la entidad de clase media, y en dicha exposición prima el componente descriptivo ya que es una categoría puesta en tensión y cuyo significado está en disputa. En segundo lugar, ambas perspectivas teóricas que disputan el sentido de esta entidad (economía y sociología) se explican exponiendo la base temática y también proponiendo al lector una pauta de lectura que anticipa las conclusiones: la adopción de la perspectiva económica para definir la clase media. Este párrafo también muestra el modelo de exposición analítica (Contursi y Ferro, 2000) en el que la idea que se quiere informar se presenta al principio del texto y existe una relación semántica entre el sujeto y el complemento de todo/parte. Es así como mediante el conector "por ejemplo" en la segunda oración introduce a las partes (sociólogos y economistas) de un todo (perspectiva del investigador) realizando el siguiente recorrido: perspectiva del investigador - sociólogos y politólogos vs economistas- adopción de perspectiva de economistas. Por otro lado, en el párrafo analizado los verbos están en tiempo presente ("suelen", "tienden", "adopta") presentando un mundo comentado en el que se exponen las perspectivas enfrentadas y la elección del enunciador, lo cual constituye un comentario, una evaluación de las perspectivas en juego, en contraposición al mundo narrado observado anteriormente que describía y explicaba históricamente el crecimiento de la clase media en América Latina.

La exposición analítica se compone de oraciones enlazadoras de fenómenos como la del siguiente ejemplo: "Como se señaló anteriormente, la movilidad tiene diferentes significados en diferentes contextos, y uno de esos significados importantes -sobre todo en un contexto intergeneracional- es el de "independencia del origen" (Ferreira y otros, 2013:25).

Nuevamente se evidencia la lógica de todo/parte (significados de 
De Prácticas y discursos

Universidad Nacional del Nordeste

Centro de Estudios Sociales

Año 7, Número 10, 2018, Octubre

ISSN 2250-6942 el "RING" Del banco MUNDIAL: ESTRATEGIAS DISCURSIVAS en el enFrentaMIENTO DE LA ECONOMÍA Y LA SOCIOLOGía PARA LA MEDICIŌN DE LA DESIGUALDAD Y MOVILIDAD SOCIAL

THE "RING" OF THE WORLD BANK: DISCURSIVE STRATEGIES IN THE CONFRONTAIION OF THE ECONOMY AND SOCIOLGGY FOR THE MEASUREMENT OF INEQUALIITY AND SOCIAL MOBILITY

movilidad/independencia de origen) con el verbo "tener", propio de la exposición analítica. Dentro los diferentes significados de movilidad social, el de independencia de origen es tomado por el texto como "importante". Este concepto que no es desarrollado en profundidad en el texto refiere al análisis de movilidad social intergeneracional independientemente del origen de su posición frente a los destinos sociales, es decir de la situación primaria del individuo (y sus antepasados, padres y abuelos), desconociendo también el origen de las desigualdades de clase.

La cita anterior está dentro del segundo momento de la secuencia lógica del género informe de investigación, el de la resolución analítica.

Este segundo momento presenta en el texto una lógica particular. El apartado "Una foto de la clase media en América Latina", por ejemplo, comienza con una serie de preguntas que el mismo texto responderá a continuación describiendo cualitativamente las características de las clases medias de la región. En este caso, el componente descriptivo se encuentra junto con el componente didáctico en una exposición que responde las preguntas planteadas al inicio del subtítulo mencionado. Estos componentes predominan en el texto ya que son centrales en la secuencia expositivo-explicativa propia del informe de investigación.

Más adelante, el subtítulo "La clase media y el contrato social" comienza de la misma forma, con preguntas a ser resueltas en una exposición que se inicia con un recorrido histórico sobre el devenir de la clase media:

Concretamente, ¿hay alguna probabilidad de que el crecimiento de la clase media de América Latina induzca a cambios en el contrato social fragmentado de la región? En un sentido amplio, un "contrato social" puede entenderse como la combinación de acuerdos implícitos y explícitos que determinan lo que cada grupo contribuye al Estado y lo que recibe de él. A grandes rasgos, el contrato social de América Latina en la segunda mitad del siglo XX se caracterizó por un Estado pequeño al que la elite (y la pequeña clase media a ella unida) contribuía con impuestos bajos y del que se beneficiaba en gran parte mediante un conjunto "truncado" de beneficios en efectivo, como pensiones de jubilación, indemnizaciones por despido, etc., a los cuales sólo tenían derecho los trabajadores formales del sector privado. (Ferreira y otros, 2013:12) 
De Prácticas y discursos

Universidad Nacional del Nordeste

Centro de Estudios Sociales

Año 7, Número 10, 2018, Octubre

ISSN 2250-6942

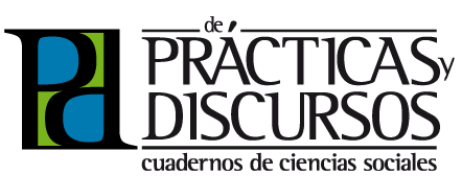

Nuevamente el texto expone en la pregunta el tema a resolver y lo explica didácticamente. Aquí observamos la coexistencia del mundo comentado y el mundo narrado (Weinreich, 1975) anteriormente nombrados. En la cita analizada se expone el concepto de contrato social desde el relato histórico, sale del comentario, de la apreciación sobre el fenómeno a explicar en presente indicativo ("puede" entenderse), y entra en la narración presentando un mundo narrado que habla del pasado con verbos en pretérito perfecto simple e imperfecto ("se caracterizó" PPS, "contribuia" $\mathrm{PI}$, "se beneficiaba" PI, "tenían" PI). Este contraste entre el mundo narrado en pasado y los verbos en presente indicativo justifica la definición de "contrato social" generando un efecto de sentido de objetividad y distancia, se presenta como una verdad universal, no una coyuntura especifica, estando en el orden del saber. A diferencia del mundo comentado, el mundo narrado borra las huellas del enunciador y presenta los hechos como sustraidos a la discusión en el presente, aumentando el efecto de objetividad con oraciones que siguen el orden lógico de sujeto-verbo-objeto. Es decir, en este contraste observamos marcas de la toma de posición del enunciador que justifica su comentario, sus definiciones sobre contrato social en este caso, sobre la definición de clases medias para sociólogos y economistas en otros, apoyándose en la historia, en un mundo narrado del cual se distancia. En la resolución analítica también se exponen algunos ejemplos que justifican la elección del índice para medir el ingreso: "Este indice es atractivo por diversos motivos: - Intuitivamente, captura los microfundamentos del crecimiento económico -a nivel de los hogares individuales. (...) • Se puede interpretar naturalmente como una medida "democrática" del crecimiento económico" (Ferreira y otros, 2013:32-33).

El rigor científico no es protagonista de estos enunciados que justifican lo "atractivo" del índice, y palabras como "intuitivamente" y "naturalmente" se apoyan sobre el sentido común que parte de una línea de pensamiento, de un conjunto de creencias que estará relacionado con el modo en que la medición de desigualdades sociales debe realizarse en vínculo con la ciencia social para justificar al enunciador. Asimismo, estos adverbios terminados en -mente manifiestan las modalidades apreciativas mediante las cuales el enunciador presenta el enunciado evaluándolo, son índices de subjetividad que exponen la postura del autor. Esta modalidad lógica bajo estos adverbios indica la 
De Prácticas y discursos

Universidad Nacional del Nordeste

Centro de Estudios Sociales

Año 7, Número 10, 2018, Octubre

ISSN 2250-6942 el "RING" Del banco MUNDIAL: ESTRATEGIAS DISCURSIVAS en el enFrentaMIENTO DE LA ECONOMÍA Y LA SOCIOLOGía PARA LA MEDICIŌN DE LA DESIGUALDAD Y MOVILIDAD SOCIAL

THE "RING" OF THE WORLD BANK: DISCURSIVE STRATEGIES IN THE CONFRONTAIION OF THE ECONOMY AND SOCIOLGGY FOR THE MEASUREMENT OF INEQUALIITY AND SOCIAL MOBILITY

\footnotetext{
${ }^{3}$ Cfr. Dubet, Francoise (2010), Les Places et les Chances. Repenser la justice sociale.
}

presencia del enunciador que entra y sale del discurso, por momentos borrándose y por otros presentándose como evaluador de perspectivas.

Los otros componentes del discurso político (prescriptivo y programático) se hacen presentes en el final evaluativo del informe, el tercer momento de la secuencia del informe de investigación, cuando por ejemplo se destacan ciertos sectores a reformar para "un contrato social más justo y más legitimo":

Incorporar el objetivo de igualdad de oportunidades más explícitamente en las políticas públicas. Esto es fundamental para asegurar que las clases medias sientan que viven en una sociedad donde esforzarse merece la pena y los méritos son recompensados en lugar de una sociedad que tiende a favorecer a los grupos privilegiados. También es fundamental para ampliar el acceso de aquellos que siguen siendo pobres o vulnerables a buenos empleos y a fuentes estables de ingresos. A pesar de que este esfuerzo requerirá reformas en una amplia gama de ámbitos, este informe pone de relieve la necesidad de mejorar la calidad de la educación pública, desde el desarrollo de habilidades cognitivas y sociales durante la primera infancia hasta la creación de mejores institutos universitarios y universidades. A su vez, una mayor igualdad de oportunidades potenciaría la eficiencia económica, contribuyendo así a abordar el persistente problema del bajo crecimiento en América Latina y mejorando las condiciones para que el sector privado en la región genere mejores y más estables empleos para todas las clases. (Ferreira y otros, 2013:13)

La "igualdad de oportunidades" como meta de políticas públicas es un concepto que ha sido largamente criticado desde la sociología ${ }^{3}$ por desconocer las desigualdades de origen de los sujetos implicados, situación que no ocurre en el campo de la economía. El párrafo analizado justifica la adopción de este objetivo exponiendo el componente prescriptivo del discurso, lo que "debe" hacerse a fin de mejorar la situación de la región, lo que es necesario universalmente: "la necesidad de mejorar la calidad de la educación pública", y el componente pragmático del mismo a través de formas verbales en infinitivo ("asegurar", "ampliar") y en futuro ("requerirá") en donde el discurso propone a futuro. Nuevamente estamos frente a una toma de posición por par- 
De Prácticas y discursos

Universidad Nacional del Nordeste

Centro de Estudios Sociales

Año 7, Número 10, 2018, Octubre

ISSN 2250-6942

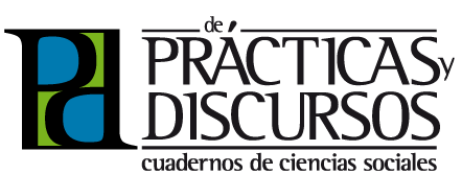

te del enunciador que se acerca a concepciones economistas y nuevamente se aleja de la sociología como ciencia para justificar su enfoque. Siendo que el enunciador es el Banco Mundial además, el componente prescriptivo toma más fuerza al saber que las recomendaciones propuestas y los conceptos elegidos son susceptibles de ser tomados por los Estados para la generación de sus propias políticas públicas.

Debemos reconocer que, si bien encontramos numerosas huellas de la elección del enunciador por una perspectiva científica ligada a la economía en el momento de evaluación del texto, esta es, sin embargo, matizada en el desarrollo argumentativo con otros términos y párrafos poco precisos que comienzan con frases como "a pesar de que en este informe no hemos llevado a cabo un análisis original (...) la literatura académica sugiere", "no hay evidencia sólida" y "es probable". Estos términos relativizan la perspectiva económica reconociendo la discusión y objeciones que la sociología puede contraponer a este desarrollo conceptual, marcando una distancia con la perspectiva económica y una nueva apertura que se relaciona con el nuevo estilo de comunicación de organismos como el Banco Mundial y el FMI que intentan alejarse del estilo más "duro" expuesto en los 90 con las "recetas neoliberales" para paises en desarrollo como fue expuesto en la introducción.

\section{TOMANDO POSICIÓN: ETHOS, EXPERTOS Y DESTINATARIOS}

Por lo expuesto hasta aquí podemos decir que la economía es la perspectiva científica privilegiada por el informe por sobre la sociología. Un análisis del ethos del discurso (cfr. Maingueneau, 2002), es decir, de la imagen del orador construida en el discurso que se instala lateralmente envolviendo a la enunciación, nos permite fundamentar esta afirmación. Si bien no es nombrado directamente, el enunciador se muestra en el discurso a partir de distintas marcas que lo van configurando, como por ejemplo en el contraste entre mundo narrado y mundo comentado explicado en el apartado anterior, en donde el enunciador se borra (mundo narrado) pero al mismo tiempo realiza una definición del contrato social tomando una perspectiva analítica por sobre otra.

En consonancia con el ejemplo anterior, el documento afirma que "a pesar de que en los siguientes capítulos aparecen a menudo los enfoques sociológicos, el informe analiza la movilidad 
De Prácticas y discursos

Universidad Nacional del Nordeste

Centro de Estudios Sociales

Año 7, Número 10, 2018, Octubre

ISSN 2250-6942 el "RING" Del banco MUNDIAL: ESTRATEGIAS DISCURSIVAS en el enFrentaMIENTO DE LA ECONOMÍA Y LA SOCIOLOGía PARA LA MEDICIŌN DE LA DESIGUALDAD Y MOVILIDAD SOCIAL

THE "RING" OF THE WORLD BANK: DISCURSIVE STRATEGIES IN THE CONFRONTAIION OF THE ECONOMY AND SOCIOLGGY FOR THE MEASUREMENT OF INEQUALIITY AND SOCIAL MOBILITY

y la dinámica de clase fundamentalmente desde una perspectiva económica" (Ferreira y otros, 2013:28). Nuevamente la elección de paradigma en el párrafo se realiza con el borramiento del enunciador, no es él quien toma la palabra y explícitamente elige, sino que es la perspectiva adoptada por "el informe", por el discurso externo a él que lo presenta objetivamente. Si bien reconoce la postura de la sociología, el conector "a pesar de que" orienta la lectura y pone el foco en la perspectiva económica, colocando a los enfoques sociológicos en una especie de paréntesis o impasse en el camino que hay que correr para poder avanzar en la lectura del informe.

Estamos frente a un ethos experto, propio del campo académico y más aún del campo de la economía, en donde la categoría del experto, que domina un solo tema o realiza una sola tarea específica, está más extendida que otras que se dedican a tareas científicas como discutir teóricamente (teórico, académico, cientista, etc). El informe se encarga de cuantificar el crecimiento de la clase media en América Latina y el Caribe, ese es su objetivo, y comienza con el capítulo de Panorámica General que estamos analizando y describe el estado actual de la teoría sobre el tema, pero esta teoría no es profundizada o discutida en el resto del documento, sino que es expuesta solo en esta primera parte ya que el objetivo como comentamos es realizar un informe más de tipo técnico.

La categoría experto es extendida en el mundo de la economía como el sujeto que realiza una tarea puntual y se acerca más al rol del técnico que al del teórico. Si bien cabría esperar que el Banco Mundial se acerque a perspectivas de la economía en su enfoque por tratarse de un banco (instituciones históricamente dominadas por economistas), la elección de definiciones economicistas de los conceptos utilizados se corresponde también con la influencia social y política que la economía como ciencia adquirió en las últimas décadas, durante las cuales "los economistas conquistaron nada menos que la capacidad de presentarse como portavoces del principio último de determinación (...) la economía se definía como un orden natural, no humano, predeterminado y determinante" (Heredia, 2015:229). En contraposición a la sociología, la Ciencia Económica se presentó "como garante de un juicio objetivo, como fundamento de un programa realista" (Heredia, 2015:25). Compitiendo con otras ciencias e incluso con las autoridades políticas, los economistas gestaron un rol de expertos a partir de la objetividad que los números. 
De Prácticas y discursos

Universidad Nacional del Nordeste

Centro de Estudios Sociales

Año 7, Número 10, 2018, Octubre

ISSN 2250-6942

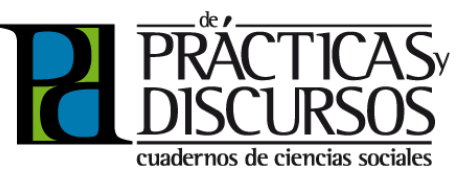

cuadernos de ciencias sociales

Este rol de experto técnico se expone en el siguiente ejemplo:

América Latina se encuentra en una encrucijada: ¿romperá (aún más) con el contrato social fragmentado que heredó de su pasado colonial y seguirá persiguiendo una mayor igualdad de oportunidades o se entregará aún más decididamente a un modelo perverso en que la clase media se excluye de participar y se vale por sí misma? Este estudio no responde a esas grandes preguntas. Se limita a plantearlas, porque se derivan naturalmente de las tendencias recientes en movilidad económica y del tamaño de la clase media (tendencias que combinan las buenas noticias del reciente aumento de los ingresos y la reducción de la pobreza con la realidad de una movilidad limitada entre generaciones y la persistencia de la desigualdad de oportunidades). Aun así, el estudio sugiere que puede que las clases medias no se conviertan automáticamente en el tan esperado agente catalítico de las reformas. (Ferreira y otros, 2013:13)

Lo que nos interesa destacar en la cita es que el informe "no responde a esas grandes preguntas", es decir, no se dedica a teorizar sobre resultados o proponer vías futuras de desarrollo, sino que "se limita" a plantearlas ya que se derivan "naturalmente" de las tendencias mostradas en el informe. Mostrar esas tendencias es justamente la tarea del experto, el objetivo del texto, evita la respuesta teórica y se centra en la respuesta técnica.

Al explicitar estas dos posturas en tensión, entre economía y sociología, retomamos la idea de que el sistema productivo del discurso se compone de gramáticas de producción, pero también de la construcción de un público, un imaginario de lectores a los que el discurso está apuntado desde la producción. Volviendo al primer nivel del discurso político planteado por Verón (1987), podemos decir que, asi como existe el ethos en el discurso político en relación con el enunciador, este tipo de discurso construye un adversario y varios tipos de destinatarios.

En primer lugar, encontramos un prodestinatario que estaría de acuerdo con la forma en que el Banco Mundial analiza y cuantifica la movilidad social utilizando criterios economicistas. Este será el destinatario ideal que imagine el documento y su objetivo será reforzar las ideas compartidas con este tipo de lector. Aqui se ubicarán los lectores que compartan la postura científica economicista del informe y acuerden con las ideas desplegadas 
De Prácticas y discursos

Universidad Nacional del Nordeste

Centro de Estudios Sociales

Año 7, Número 10, 2018, Octubre

ISSN 2250-6942 el "RING" Del banco MUNDIAL: ESTRATEGIAS DISCURSIVAS en el enFrentaMIENTO DE LA ECONOMÍA Y La SOCIOLOGİA PARA LA MEDICIŌN DE LA DESIGUALDAD Y MOVILIDAD SOCIAL

THE "RING" OF THE WORLD BANK: DISCURSIVE STRATEGIES IN THE CONFRONTAIION OF THE ECONOMY AND SOCIOLGGY FOR THE MEASUREMENT OF INEQUALIITY AND SOCIAL MOBILITY

por el documento. Un "nosotros" inclusivo es el que marcará la inscripción de este tipo de destinatario. No lo encontramos explicitamente en el texto ya que siempre se habla poniendo como sujeto de la enunciación al informe: "este informe toma", "el informe destaca", etc.

Por otro lado, la producción del discurso construye un antidestinatario, el adversario, los lectores que estarán totalmente en desacuerdo con las elecciones y los postulados presentes en el informe del Banco Mundial. Dentro de este grupo de lectores podemos ubicar a los lectores cercanos a las ideas de la sociología, campo que el discurso analizado nombra como enfrentado a la economía. El discurso del Banco Mundial parece construir a los sociólogos como antidestinatarios, ya que enfrenta ambos campos continuamente (como describimos más arriba). Al ponerse del lado de los economistas, lógicamente el adversario quedaría construido por el campo de la sociología.

Por último, el informe del Banco Mundial erige también un paradestinatario, conformado por los lectores cuyo objetivo es persuadir. El documento parece dialogar constantemente con este tipo de lector en el apartado analizado, ya que expone y contrasta las dos posturas antes descritas, intentando explicitar lo beneficioso de las elecciones realizadas por el informe. Las entidades enumerables referidas a los conceptos utilizados, la clase media y la pobreza, y las relacionadas a las posturas enfrentadas, economistas y sociólogos, se orientan a convencer a estos lectores. Dentro de este tipo de destinatarios del informe del Banco Mundial podrian estar, además de cientistas sociales todavía indecisos en el paradigma a seguir para el análisis de estratificación social, los Estados y partidos políticos que utilizan los resultados de este tipo de publicaciones para justificar decisiones politicas o afirmar sus gestiones. A esto nos referíamos cuando comentamos el componente didáctico del discurso y el componente prescriptivo.

\section{REFLEXIONES FINALES}

El apartado de Panorámica General que analizamos está compuesto por los comentarios, definiciones y discusiones preliminares del informe del Banco Mundial que en las páginas consecuentes se dedica a analizar y justificar más específicamente las decisiones metodológicas y teóricas tomadas para realizar este 
De Prácticas y discursos

Universidad Nacional del Nordeste

Centro de Estudios Sociales

Año 7, Número 10, 2018, Octubre

ISSN 2250-6942

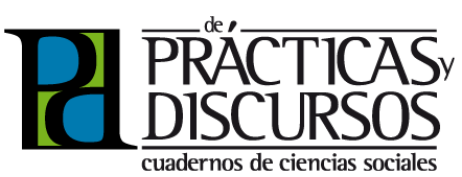

estudio. Este apartado funciona dentro del informe total como anticipo del análisis, que expone las decisiones tomadas y las discusiones teóricas que esa elección atravesó para llegar a una resolución que permita comenzar el análisis técnico.

Luego del análisis del discurso realizado podemos afirmar que la perspectiva economicista es la privilegiada en el informe, pero la misma es matizada con definiciones sociológicas y referencias a la parcialidad de este paradigma que en todo el texto intenta presentarse como conciliador y alejarse de su pasado neoliberal. La relación entre la visión economicista y la ideología neoliberal ha sido nombrada en este trabajo, pero no desarrollada en profundidad, lo que puede ser una interesante línea de investigación a futuro. En una época en la que los organismos internacionales son fuertemente cuestionados luego del fracaso de las recetas neoliberales impuestas por el Consenso de Washington para países de Latinoamérica y el Caribe, la objetividad de este tipo de informes se hace necesaria para sostener cierta credibilidad y exponerse como organismos supranacionales que velan por el bienestar mundial, sin intereses económicos ulteriores como los que fueran denunciados por Joseph Stiglitz. Esta objetividad es perseguida por el informe en el contraste entre mundo comentado y mundo narrado, el ethos experto y otras marcas de subjetividad analizadas anteriormente que intentan borrar al enunciador. La pretensión de objetividad del informe sumado a su carácter de discurso político genera un discurso de estilo conciliador en donde el enunciador intenta borrarse al evaluar y elegir conceptos y medidas recordándonos que, a pesar de su parcialidad, la economía como campo será la medida correcta, y que el mejoramiento del "contrato social" a partir de las recomendaciones del Banco Mundial será el camino para mejorar la movilidad social ascendente en la región. El rol "recomendador" de la institución en cuestión está presente a lo largo de todo el texto, pero para que el Banco Mundial sea escuchado nuevamente por los paises latinoamericanos que se vieron empobrecidos luego de seguir sus consejos en la década de 1990 deberá conciliar intereses, acercar posiciones y presentarse de forma atractiva para el trabajo conjunto con estos Estados. Este cambio en el estilo consideramos que está orientado a persuadir a un paradestinatario que ya ha sido decepcionado por los organismos internacionales de financiamiento.

El objetivo de este trabajo fue develar el posicionamiento político 
De Prácticas y discursos

Universidad Nacional del Nordeste

Centro de Estudios Sociales

Año 7, Número 10, 2018, Octubre

ISSN 2250-6942 el "RING" Del banco MUNDIAL: ESTRATEGIAS DISCURSIVAS en el enFrentaMIENTO DE LA ECONOMÍA Y LA SOCIOLOGÍA PARA LA MEDICIÓN DE LA DESIGUALDAD Y MOVILIDAD SOCIAL

THE "RING" OF THE WORLD BANK: DISCURSIVE STRATEGIES IN THE CONFRONTATION OF THE ECONOMY AND SOCIOLOGY FOR THE MEASUREMENT OF INEQUALLITY AND SOCIAL MOBILITY

académico que el Banco Mundial tiene en referencia a nuestro campo de estudio más general, la estructura y movilidad social, para, desde la sociología, poder discutir y realizar intercambios enriquecedores con dicho organismo. Al reconocernos (nuestro equipo de investigación) como el contradestinatario de dicho discurso, no nos sorprende encontrar ciertas categorizaciones y elecciones metodológicas como polémicas, economicistas y cargadas de un bagaje político que tendremos que seguir desdeñando. Este primer paso a partir del análisis del discurso nos permite situarnos y situar a nuestro "adversario" para, desde el mismo campo semántico y siguiendo la lógica isotópica expuesta, poder enfrentarnos con esas posturas y hacer crecer este campo de investigación. El ring ya está planteado y el adversario también, queda en nosotros comenzar esta contienda académica que evidentemente se dará a partir del discurso, la única materialidad que nuestras ideas pueden tener.

\section{BIBLIOGRAFÍA}

AMOSSY, R. (2000). L'argumentation dans le discours. Discourse politique, littérature d'idées, fiction, fiction. Paris: Nathan.

ANGENOT, M. (1982). La parole plamphlétaire. Typologie des discours modernes. París: Payot.

ARNOUX, E. (2006). Análisis del discurso. Modos de abordar materiales de archivo, Cap. 1, 2 y 3. Buenos Aires: Santiago Arcos.

BAJTíN, M. (1979). Estética de la creación verbal, México-Madrid-Buenos Aires-Bogotá: Siglo XXI, 1985. Comisión Económica para América Latina y el Caribe (Cepal) y Naciones Unidas (1996). Quince años de desempeño económico. América Latina y el Caribe 1980-1995. México DF: Fondo de Cultura Económica.

CONTURSI, M.E. Y FERRO, F. (2000). Los géneros académicos. Buenos Aires: Prociencia.

CHANG, H.J. (2004). Retirar la escalera. La estrategia del desarrollo en perspectiva histórica. Madrid: Libros de la Catarata.

HEREDIA, M. (2015). Cuando los economistas tomaron el poder (o cómo se gestó la confianza en los expertos). Buenos Aires: Siglo XXI. 
KERBRAT-ORECCHIONI, C. (1981). La enunciación. De la subjetividad en el lenguaje. Buenos Aires: Edicial, 1993.

LACLAU, E. (1995). Emancipación y diferencia. Barcelona: Ariel. Mangone, C. y Warley, J. (1994). El discurso político, del foro a la televisión. Buenos Aires: Biblos.

PECHEUX, M. (1978). Hacia un análisis automático del discurso,. Madrid: Gredos.

PERRET, D. (1970). Les appellatifs. Analyse lexicale et actes de parole. Langages, 17.

STIGLITZ, J. (2002). El malestar en la globalización. Madrid: Taurus.

STIGLITZ, J. (2012). El precio de la desigualdad. Madrid: Taurus. Disponible en: http://depa.fquim.unam.mx/amyd/archivero/ EPreciodelaDesigualdad_27245.pdf

VERÓN, E. (1988). Prensa gráfica y teoria de los discursos sociales: producción, recepción, regulación. En Verón, E. (2004). Fragmentos de un discurso. Barcelona: Ed. Gedisa.

VERÓN, E. (1987). La palabra adversativa. Observaciones sobre la enunciación política. En Verón, E. et al., El discurso político. Lenguajes y acontecimientos (pp. 11-26). Buenos Aires: Hachette.

WILLIAMSON, J. (2003). No hay consenso. Reseña sobre el Consenso de Washington y sugerencias sobre los pasos a dar. Finanzas y Desarrollo. Washington: Fondo Monetario Internacional.

WEINREICH, U. (1975). Mundo comentado/mundo narrado. En Estructura y función de los tiempos en el lenguaje. Madrid: Gredos.

\section{CORPUS}

FERREIRA, F.H.G.; MESSINA, J.; RIGOLINI, J.; LÓPEZ-CALVA, L.F.; LUGO, M.A. Y RENOS VAKIS (2013). La movilidad económica y el crecimiento de la clase media en América Latina (pp. 19-35). Washington, DC: Banco Mundial. Licencia: Creative Commons de Reconocimiento CC BY 3.0 Panorámica General. 